\title{
EL USO DE MATERIAS PRIMAS LÍTICAS A TRAVÉS DEL TIEMPO EN SEIS LOCALIDADES DE LA COSTA NORTE DEL GOLFO SAN MATÍAS (RÍO NEGRO, ARGENTINA)
}

\author{
USE OF LITHIC RAW MATERIAL OVER TIME IN SIX ARCHAEOLOGICAL \\ LOCALITIES ON THE NORTHERN COAST OF THE SAN MATÍAS GULF \\ (RÍO NEGRO, ARGENTINA)
}

\begin{abstract}
Jimena Alberti ${ }^{1}$
La costa rionegrina del Golfo San Matías presenta ocupaciones de cazadores-recolectores-pescadores desde el Holoceno Medio hasta el Tardío. Durante ese período temporal se registra el consumo de recursos tanto marinos como terrestres, y la manufactura de una amplia variabilidad de artefactos líticos, utilizando principalmente cuatro tipos de rocas a lo largo del tiempo. En investigaciones previas se realizaron estudios del uso de las materias primas a nivel regional efectuando comparaciones entre diferentes sectores del golfo. En este trabajo la escala de observación se centra en seis localidades particulares, las cuales presentan fechados tanto del bloque temprano de ocupación como del tardío. Esto se realizó con el objetivo de discutir los cambios a través del tiempo al interior de cada conjunto, y de evaluar si estas pequeñas escalas continúan las tendencias regionales determinadas en otros trabajos. Se desarrollaron análisis de materias primas y tecnológicos y se aplicaron métodos estadísticos para determinar tendencias generales en las muestras. Los resultados indican que, a diferencia de lo planteado anteriormente, los tipos de rocas usadas cambiaron a lo largo del tiempo y que algunas de las localidades estudiadas presentaron también diferencias en las restantes variables analizadas (porcentaje de corteza, artefactos enteros vs. fragmentados, entre otras).
\end{abstract}

Palabras claves: tecnología lítica, cazadores-recolectores, ocupaciones costeras, Holoceno Medio y Tardío.

The coast of the Rio Negro province presents hunter-gatherer-fisher occupations dated from the Middle to the Late Holocene, a period in which the utilization of marine and terrestrial resources is recorded, as well as the manufacturing of a wide variety of lithic artifacts using mainly four types of rocks. In previous papers, we studied the use of lithic raw materials at a regional level, comparing different areas of the San Matías Gulf. In this paper, the scale of analysis focuses on six specific archaeological localities, which are dated through the entire human occupation of the area. The aim is to analyze changes over time within each set of lithic materials, and to determine if the scales continue to show the regional trends established in previous papers. Technological and raw material analyses were performed, and statistical methods were applied to establish general trends in the samples. The results indicate that, unlike previous studies, the type of rocks that were used varied over time, and that several of the studied localities also show significant variations in the other indicators that were analyzed (cortex percentage, broken vs. unbroken artifacts, among others).

Key words: Lithic technology, hunter-gatherers, coastal occupations, Middle and Late Holocene.

El estudio de la tecnología lítica constituye una de las herramientas existentes para el entendimiento de los modos de vida de las poblaciones del pasado. Un punto de partida fundamental para este estudio es la determinación de las fuentes de materia prima lítica existentes en un área determinada y el establecimiento de cómo esas fuentes pudieron haber sido explotadas y aprovechadas en el pasado(Andrefsky 1994; Becketal. 2002; Ericson 1984; Torrence 1983; entre otros). En investigaciones previas (Alberti 2016; Alberti y Cardillo 2015; Alberti et al. 2015;
Favier Dubois y Alberti 2014) se delimitó la base regional de recursos líticos (sensu Ericson 1984) de la costa del golfo San Matías (provincia de Río Negro) a partir de la utilización de las hojas geológicas regionales, la consulta de bibliografía específica y el trabajo de relevamiento en el campo. El uso conjunto de estas herramientas permitió determinar la existencia de fuentes primarias y secundarias en el área, compuestas por diferentes tipos de rocas y, a partir de ello, estudiar, la explotación de rocas a nivel regional, estableciéndose tendencias particulares para

\footnotetext{
${ }^{1}$ Instituto Multidisciplinario de Historia y Ciencias Humanas (IMHICIHU), Consejo Nacional de Investigaciones Científicas y Técnicas (CONICET), Buenos Aires, Argentina. jimealberti@ gmail.com
}

Recibido: abril 2019. Aceptado: agosto 2019.

http://dx.doi.org/10.4067/S0717-73562020005001003. Publicado en línea: 22-junio-2020. 
los diferentes momentos de ocupación del área (Alberti 2016). Esta comparación, tal como se ha dicho, se realizó a nivel regional, sin discriminar localidades. Así, el objetivo de este trabajo es poner a prueba estos primeros resultados a partir del análisis de los conjuntos de artefactos recuperados en seis localidades de la costa rionegrina, las cuales presentan ocupaciones de grupos cazadoresrecolectores-pescadores datadas en ambos bloques temporales de consumo de recursos marinos en el área (ver más adelante). En trabajos previos (Alberti 2016) fueron realizadas subdivisiones temporales al interior de cada bloque con el objetivo de reducir el amplio lapso abarcado por cada uno de ellos y testear posibles diferencias en las tendencias a lo largo del tiempo. Sin embargo, esto no arrojó resultados significativos, por lo que en este trabajo se decidió retomar las divisiones originalmente propuestas en Favier Dubois et al. (2009). Entonces, los análisis aquí desarrollados aportan al conocimiento del uso particular de ciertos sectores del espacio de la costa rionegrina, más allá de las tendencias regionales ya establecidas. Esta nueva información permitirá comenzar a abordar temas como la intensidad de ocupación de las localidades, los rangos de movilidad de los grupos y la explotación de diferentes recursos en distintos sectores del espacio, entre otros.

\section{Contexto Regional y Antecedentes}

La costa norte del golfo San Matías se extiende entre la ciudad de San Antonio Oeste (SAO) y la desembocadura del río Negro en el Atlántico (Figura 1). Allí se alternan cordones medanosos, bajos, playas y acantilados (Gonzalez Díaz y Malagnino 1984). Esta costa sufrió cambios en su configuración a lo largo del Holoceno que impactaron en la ocupación humana de este espacio y en la preservación del registro arqueológico (p.ej., Favier Dubois 2013; Favier Dubois et al. 2016). En el primer pulso de descenso y estabilidad del nivel del mar al que refieren los trabajos geológicos en Patagonia (i.e. Codignotto et al. 1987; Monti 2000; Schellmann y Radtke 2010), entre $6500 / 6000$ y 3800/3200 ${ }^{14} \mathrm{C} \mathrm{AP}$, se pudo haber dado el inicio del relleno de los entrantes marinos (Favier Dubois 2013). Luego del $3200{ }^{14} \mathrm{C} \mathrm{AP}$, con el segundo pulso, habría comenzado la acreción costera en una escala más amplia, dando lugar a la formación de cordones litorales los cuales habrían funcionado como fuentes de recursos líticos para los momentos tardíos de la ocupación litoral (Favier Dubois 2013; Favier Dubois y Kokot 2011; Favier Dubois y Scartascini 2012), ampliando la oferta de rocas disponibles, que previamente habría sido escasa con muy poca presencia de fuentes secundarias (principalmente depósitos de guijarros en forma de cordones litorales sobre las paleoplayas) (Alberti 2012, 2016; Favier Dubois y Alberti 2014).

Teniendo en cuenta estos cambios en la disponibilidad de rocas a lo largo del Holoceno, en trabajos anteriores se estudió el uso de las materias primas líticas desde una perspectiva regional, agrupando las localidades arqueológicas por bloque temporal de
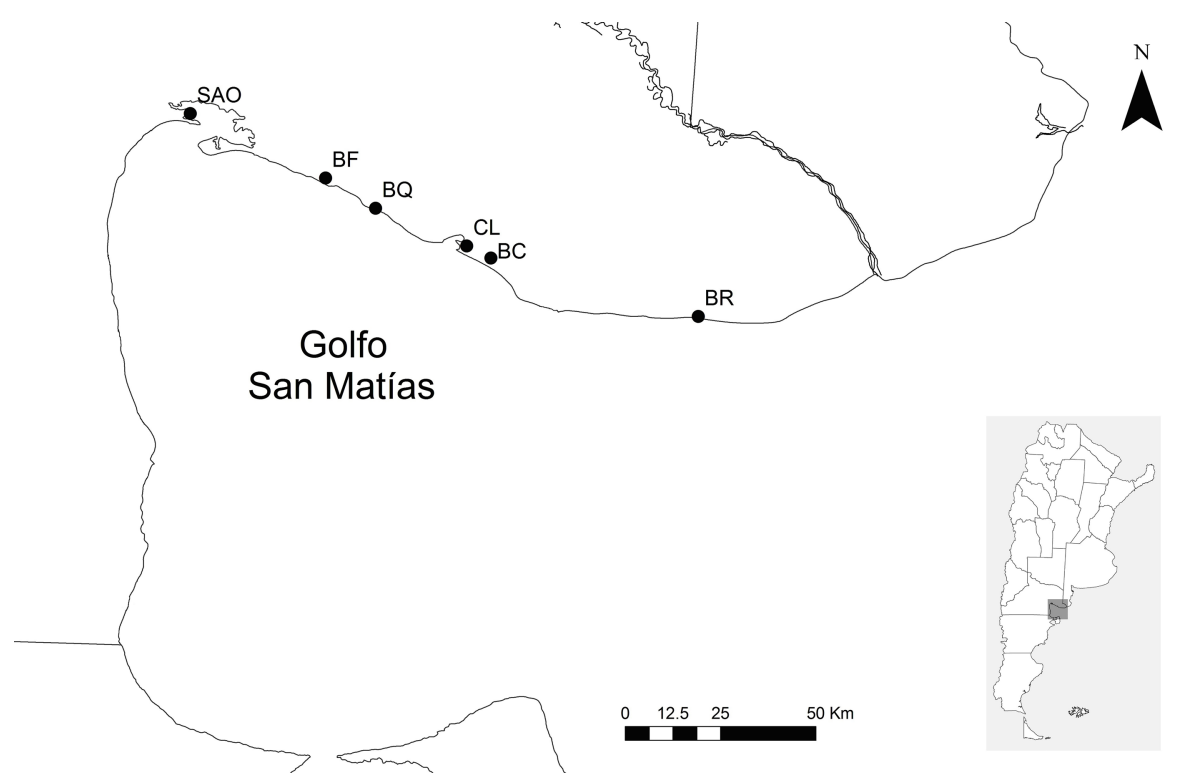

Figura 1. Golfo San Matías, provincia de Río Negro. Se señalan las localidades tomadas para su estudio en este trabajo (ver más adelante). SAO: San Antonio Oeste. BF: Bahía Final. BQ: Bajo de la Quinta. CL: Caleta de los Loros. BC: Bahía Creek. BR: Bahía Rosas. 
consumo de recursos (Alberti 2016). Dichos bloques se vinculan con un consumo intensivo de recursos marinos para los inicios de la ocupación del golfo (desde los 6000 años ${ }^{14} \mathrm{C}$ AP en adelante) y el incremento del consumo de recursos terrestres en la dieta desde los ca. 1500 años ${ }^{14} \mathrm{C}$ AP (Favier Dubois et al. 2009; Favier Dubois y Scartascini 2012; Scartascini 2014). Los resultados obtenidos en estos estudios sugieren que, pese a la existencia de cambios en la línea costera mencionados más arriba, el uso de las rocas a lo largo del tiempo no varió de manera significativa (Alberti 2016). Es decir, el uso de materias primas predominantemente no locales que se registra para los inicios de la ocupación del golfo (rocas silíceas y calcedonia, principalmente) continuó hasta el Holoceno Tardío, pese a la disponibilidad media de rocas de buena calidad para la talla existente para momentos tardíos, como son las volcanitas básicas.

Para los nuevos análisis presentados en este trabajo, las localidades tomadas fueron San Antonio Oeste (SAO), Bahía Final (BF), Bajo de la Quinta (BQ), Caleta de los Loros (CL), Bahía Creek (BC) y Bahía Rosas (BR) (Figura 1). Todas ellas constituyen sitios a cielo abierto en los cuales los materiales se presentan en contexto de superficie y, en algunos casos, en concheros. Estos últimos no fueron incluidos en este trabajo ya que implican un abordaje metodológico diferente al de los materiales de superficie. En el caso de BF se consideraron otras concentraciones dentro de la misma localidad, distintas a las analizadas previamente (Alberti 2012). Las asignaciones temporales de las muestras fueron realizadas a partir de la obtención de fechados directos de materiales asociados a los artefactos líticos o de criterios geomorfológicos según la ubicación de los loci en el paisaje.

\section{Metodología de Trabajo}

La metodología general de trabajo utilizada para el análisis de las fuentes de materias primas del área de estudio ha sido descrita en trabajos previos (remitirse a Alberti 2012, 2016), con lo que acá únicamente realizaremos una breve mención. Para este relevamiento se utilizaron las hojas geológicas del área en combinación con la información geomorfológica disponible (Etcheverría et al. 2006; Gelós et al. 1988, 1990; Martinez et al. 2001), y se realizaron muestreos en el campo utilizando una metodología distribucional a partir del establecimiento de transectas y de la realización de muestreos sistemáticos por área y por tiempo (Franco y Borrero 1999). Las transectas fueron realizadas por dos personas, registrando la cantidad de nódulos encontrados y el tiempo y distancia recorridos. En algunos casos el material fue recolectado para ser llevado al laboratorio, mientras que en otros solamente se tomaron los datos en el campo mediante planillas confeccionadas para tal fin. En los muestreos por área, los tamaños de las cuadrículas variaron dependiendo de la densidad de material encontrado. Para clasificar la disponibilidad de materia prima lítica se tomaron los criterios establecidos por Meltzer (1989) y modificados por Civalero y Franco (2003). El primer autor establece que las rocas localizadas en distancias de hasta $40 \mathrm{~km}$ del sitio son locales, mientras que las no locales se ubican más allá de esa distancia. Por su parte, Civalero y Franco (2003) establecen más divisiones de acuerdo con las distancias: más de $40 \mathrm{~km}$ desde el sitio es no local, entre 40 y $10 \mathrm{~km}$ es local y menos de $5 \mathrm{~km}$ es inmediatamente disponible. En el laboratorio se determinaron macroscópicamente los tipos de materias primas (sensu Alberti y Fernández 2015) utilizando la litoteca de referencia construida para el área y luego se seleccionaron algunos tipos característicos de mayor representatividad en las fuentes para ser identificados mediante cortes delgados. La calidad de las rocas para la talla se estableció siguiendo a Aragón y Franco (1997). También se determinó la forma de los nódulos (según Zingg 1935 en Shelley 1993) y se registraron las variables métricas (tamaño, peso y volumen) de cada uno de ellos. Esta información no se incluye en este trabajo por no resultar directamente pertinente, pero está disponible para consulta en otras publicaciones (p.ej., Alberti 2016).

Para la recolección de artefactos en las localidades arqueológicas se realizaron muestreos sistemáticos de forma aleatoria y con recolección en cuadrículas de tamaño variable (de entre 4 y $25 \mathrm{~m}^{2}$ ) según la densidad artefactual de cada sector, siguiendo también una metodología de tipo distribucional (Franco y Borrero 1999). En el laboratorio se determinaron los tipos de materias primas líticas utilizadas para la manufactura y se registraron las variables métricas de los artefactos. La caracterización tecno-morfológica del conjunto se realizó según Aschero $(1975,1983)$ registrándose tipo de artefacto (núcleos, lascas, filos naturales con rastros complementarios e instrumentos), estado del artefacto (entero o fragmentado) y porcentaje de corteza presente en la cara dorsal (sensu Franco 2002). Para evitar sobrerepresentaciones, fueron tomadas únicamente las lascas enteras y fracturadas con talón.

\section{Análisis estadísticos}

La frecuencia y la composición son atributos que pueden ser utilizados para describir los conjuntos arqueológicos. La frecuencia es la cantidad de veces que cierta clase de artefactos aparece en el conjunto, mientras que la composición tiene que ver con la cantidad de clases que componen dicho conjunto. Para la comparación de las muestras se realizó un contraste de hipótesis $\left(\mathrm{X}^{2}\right)$, cuyo objetivo es comprobar si una 
diferencia observada es real o producto de una variación aleatoria, para lo cual se calculan las diferencias entre las frecuencias observadas y las esperadas (Freedman et al. 1993; Moore 1995). Valores elevados indican que las observadas están muy alejadas de las esperadas y viceversa (Freedman et al. 1993, Moore, 1995).

La relación entre la media y la varianza de una muestra implica, generalmente, que conjuntos que tengan frecuencias mayores van a tener una varianza mayor. $\mathrm{Al}$ usarse distancias como la Euclideana, las ausencias en las matrices de datos (consignadas como 0 ) son interpretadas como similitudes y no como faltas, lo cual implica que los conjuntos más pequeños se agrupen sobre la base de la ausencia de rasgos más que por su diversidad tipológica. Como resultado, la variación se ve disminuida. Para reducir este efecto se utilizó la distancia Euclideana con los datos (clases) transformados a proporciones (Borcard et al. 2011; Legendre y Legendre 1998). Así, en este trabajo cada conjunto de la muestra $(\mathrm{n}=12$, dos conjuntos por cada localidad muestreada: uno perteneciente a los materiales del bloque temprano y otro a los del tardío) representa uno o más muestreos realizados sobre los sitios. Para describir cada una de estas unidades se generaron diferentes variables: tipos de artefactos, tipos de rocas (las cuatro más abundantes: volcanitas ácidas e intermedias-básicas, sílex y calcedonia), estado de los artefactos, porcentaje de corteza y localización de cada loci (expresada mediante coordenadas). Cada conjunto de datos posee diferentes niveles de homogeneidad interna, influenciada por la relación entre la diversidad y el tamaño de las muestras. En este contexto, la aplicación de análisis multifactoriales puede ser una herramienta exploratoria y analítica, dado que permite la descripción simultánea de distintos conjuntos de variables (como materias primas o clases artefactuales). Esto balancea la influencia de estos conjuntos y permite comparaciones directas (Borcard et al. 2011; Escoffier y Pagès 1998; Pagès 2002). Así, se realizó un análisis factorial múltiple (AFM) en el que cada grupo de variables es analizado independientemente mediante un análisis de componentes principales (CP). El CP permite extraer las tendencias generales de variación a partir de la correlación lineal de cada variable transformada. Posteriormente, cada conjunto de variables obtenidas mediante este procedimiento es estandarizado de acuerdo con el primer autovalor (el más alto) de cada grupo (Escoffier y Pagès 1998; Pagès 2002). La estandarización es necesaria debido a que, si todos los conjuntos de variables son introducidos como elementos activos, uno solo de ellos puede sobre-representar los resultados del primer eje. Al usar la varianza máxima de los ejes para estandarizar cada grupo de variables, el análisis global balancea cada contribución de acuerdo con la variación total, con lo que la estructura de cada conjunto es respetada (Escoffier y Pagès 1998; Pagès 2002). Este tipo de análisis es correlativo porque excluye la posibilidad de la influencia de unos grupos de datos sobre otros (Borcard et al. 2011), dejando abierta la posibilidad de que una o más variables puedan ser incorporadas a posteriori en el test, como es el caso de la localización de los conjuntos en el espacio. La correlación entre las variables originales y los nuevos ejes puede ser representada de manera gráfica o numérica. Para la realización de todos los análisis fueron utilizados los programas R 2.5.1 (R Development Core Team 2005) y PAST (Hammer et al. 2001).

\section{Resultados}

\section{San Antonio Oeste}

La muestra total recuperada es de 1.352 artefactos (Tabla 1). Tal como se desprende de la Tabla 1, el mayor

Tabla 1. San Antonio Oeste. Cantidad de artefactos divididos por las rocas más utilizadas.

San Antonio Oeste. Lithic artifacts divided by most used rocks.

\begin{tabular}{|c|c|c|c|c|c|}
\hline \multirow{2}{*}{ Bloque Antiguo } & \multicolumn{3}{|c|}{ Clase Artefactual } & \multirow{2}{*}{$\begin{array}{l}\text { Subtotal } \\
\text { MP (n) }\end{array}$} & \multirow{2}{*}{$\begin{array}{l}\text { Subtotal } \\
\text { MP (\%) }\end{array}$} \\
\hline & Lascas & Núcleos & Instrumentos & & \\
\hline Volcanitas intermedias-básicas & 422 & 34 & 18 & 474 & 36,92 \\
\hline Sílex & 406 & 44 & 13 & 463 & 36,06 \\
\hline Calcedonia & 202 & 13 & 5 & 220 & 17,13 \\
\hline Volcanitas ácidas & 87 & 26 & 14 & 127 & 9,89 \\
\hline Subtotal clase de artefacto (n) & 1.117 & 117 & 50 & 1.284 & 100 \\
\hline Subtotal clase de artefacto $(\%)$ & 86,99 & 9,11 & 3,89 & 100 & \\
\hline \multicolumn{6}{|l|}{ Bloque Reciente } \\
\hline Calcedonia & 16 & 0 & 3 & 19 & 27,94 \\
\hline Sílex & 13 & 3 & 1 & 17 & 25,00 \\
\hline Volcanitas intermedias-básicas & 15 & 1 & 1 & 17 & 25,00 \\
\hline Volcanitas ácidas & 10 & 4 & 1 & 15 & 22,06 \\
\hline Subtotal clase de artefacto (n) & 54 & 8 & 6 & 68 & 100 \\
\hline Subtotal clase de artefacto $(\%)$ & 79,41 & 11,76 & 8,82 & 100 & \\
\hline
\end{tabular}


porcentaje de artefactos corresponde a lascas, seguido por los instrumentos y los núcleos en tercer lugar. En cuanto a las materias primas, la distribución de las frecuencias sugiere diferencias a lo largo del tiempo. Mientras en el bloque antiguo se utilizaron mayormente volcanitas intermedias-básicas, estas rocas caen al tercer lugar en el bloque tardío, intercambiando su lugar con la calcedonia. Estas diferencias detectadas resultaron ser estadísticamente significativas $\left(\mathrm{X}^{2}=18,12\right.$, $\mathrm{p}$-valor $\left.<0,01\right)$ con lo que se puede afirmar que la variación en el uso de las rocas no respondería a factores relacionados con el azar, sino que tendría que ver con otras causas.
Otra de las variables consignadas fue el porcentaje de corteza presente en la cara dorsal por materia prima (Tabla 2). En ambos casos predominan los artefactos con $0 \%$ de corteza, seguidos de los artefactos con $100 \%$ de corteza. Sin embargo, en el bloque reciente los artefactos con 100\% de corteza aparecen en mayor cantidad que en el bloque previo.

La última variable consignada fue la cantidad y proporción de artefactos enteros por materia prima y por bloque temporal. En este sentido, los resultados (Tabla 2) muestran que las proporciones de artefactos enteros $\mathrm{y}$ fragmentados son similares en ambos bloques; sin

Tabla 2. San Atonio Oeste. Cantidad y porcentaje de artefactos con diferentes porcentajes de corteza y estado, divididos por materia prima. E: enteros. Fg: fragmentados.

San antonio Oeste. Lithic artifacts with different percentages of cortex and condition, divided by raw material. E: unbroken. Fg: broken.

\begin{tabular}{lcccccccc}
\hline \multirow{2}{*}{ Bloque Antiguo } & \multicolumn{3}{c}{ Porcentaje de Corteza } & \multicolumn{3}{c}{ Estado } \\
\cline { 2 - 9 } & 0 & 25 & 50 & 75 & 100 & E & Fg \\
\hline Volcanitas intermedias-básicas & 349 & 22 & 23 & 22 & 58 & 244 & 230 \\
\hline Sílex & 335 & 38 & 17 & 31 & 42 & 225 & 238 \\
\hline Calcedonia & 180 & 8 & 5 & 11 & 16 & 110 & 110 \\
\hline Volcanitas ácidas & 61 & 7 & 6 & 15 & 38 & 73 & 54 \\
\hline \multicolumn{1}{c}{ Total porcentaje de corteza y estado (n) } & 925 & 75 & 51 & 79 & 154 & 652 & 632 \\
\hline \multicolumn{1}{c}{ Total porcentaje de corteza y estado $(\%)$} & 72,04 & 5,84 & 3,97 & 6,15 & 11,99 & 50,78 & 49,22 \\
\hline Bloque Reciente & & & & & & & \\
\hline Calcedonia & 17 & 1 & 0 & 0 & 1 & 11 & 8 \\
\hline Sílex & 11 & 4 & 1 & 1 & 0 & 9 & 8 \\
\hline Volcanitas intermedias-básicas & 8 & 2 & 0 & 1 & 6 & 6 & 11 \\
\hline Volcanitas ácidas & 6 & 0 & 2 & 4 & 3 & 7 & 8 \\
\hline Total porcentaje de corteza y estado (n) & 42 & 7 & 3 & 6 & 10 & 33 & 35 \\
\hline Total porcentaje de corteza y estado $(\%)$ & 61,8 & 10 & 4,4 & 8,8 & 14,7 & 48,53 & 51,47 \\
\hline
\end{tabular}

embargo en el bloque antiguo hay ligeramente mayor proporción de artefactos enteros, mientras que en el reciente ocurre lo contrario. En este caso se testeó mediante $\mathrm{X}^{2}$ los porcentajes de artefactos enteros y fragmentados. Los resultados indicaron que no hay diferencias estadísticamente significativas entre ambas muestras $\left(\mathrm{X}^{2}=0,13\right.$, $\mathrm{p}$-valor $\left.>0,05\right)$.

\section{Bahía Final}

En esta localidad se recuperaron 391 artefactos (Tabla 3). En esta muestra también predominan los desechos por sobre el resto de las categorías, pero hay diferencias entre ambos bloques. En el antiguo la proporción de lascas es menor que en el reciente, mientras que con los instrumentos sucede lo contrario. A su vez, la proporción de núcleos en el bloque reciente aumenta. Otra diferencia entre ambos bloques son las materias primas que fueron utilizadas. Mientras que en el antiguo las volcanitas intermedias-básicas ocupan el primer lugar, en el reciente caen al tercero. El sílex, a su vez, pasa del segundo lugar en el bloque antiguo, al primero en el reciente. Finalmente, el jaspe, que aparece en cuarto lugar de importancia en el bloque antiguo, en el reciente desaparece y es reemplazado por las volcanitas ácidas. Estas diferencias resultaron ser estadísticamente significativas $\left(X^{2}=19,31\right.$, p-valor $<0,01$ ).

Para el porcentaje de corteza, los datos se distribuyeron como se muestra en la Tabla 4. En esta variable se registran cambios en uno y otro bloque temporal. Mientras que en el antiguo predominan los artefactos con $0 \%$ de corteza, seguidos de los que tienen $25 \%$, en el reciente predominan también los que tienen $0 \%$ de corteza, pero el segundo lugar lo ocupan los artefactos con $100 \%$ de corteza.

En la última variable analizada (Tabla 4) también los valores son distintos para ambos bloques: mientras que en el antiguo hay más artefactos fragmentados 
Tabla 3. Bahía Final. Cantidad de artefactos divididos por las rocas más utilizadas.

Bahía Final. Lithic artifacts divided by most used rocks.

\begin{tabular}{|c|c|c|c|c|c|}
\hline \multirow{2}{*}{ Bloque Antiguo } & \multicolumn{3}{|c|}{ Clase Artefactual } & \multirow{2}{*}{$\begin{array}{l}\text { Subtotal MP } \\
\text { (n) }\end{array}$} & \multirow{2}{*}{$\begin{array}{l}\text { Subtotal MP } \\
(\%)\end{array}$} \\
\hline & Lascas & Núcleos & Instrumentos & & \\
\hline Volcanitas intermedias-básicas & 73 & 8 & 2 & 83 & 43,68 \\
\hline Sílex & 48 & 9 & 6 & 63 & 33,16 \\
\hline Calcedonia & 28 & 2 & 6 & 36 & 18.95 \\
\hline Jaspe & 8 & 0 & 0 & 8 & 4,21 \\
\hline Subtotal clase de artefacto (n) & 157 & 19 & 14 & 190 & 100 \\
\hline Subtotal clase de artefacto (\%) & 82,63 & 10 & 7,37 & 100 & \\
\hline \multicolumn{6}{|l|}{ Bloque Reciente } \\
\hline Sílex & 74 & 14 & 0 & 88 & 43,78 \\
\hline Calcedonia & 51 & 8 & 0 & 59 & 29,35 \\
\hline Volcanitas intermedias-básicas & 40 & 5 & 2 & 47 & 23,38 \\
\hline Volcanitas ácidas & 7 & 0 & 0 & 7 & 3,48 \\
\hline Subtotal clase de artefacto (n) & 172 & 27 & 2 & 201 & 100 \\
\hline Subtotal clase de artefacto (\%) & 85,57 & 13,43 & 1 & 100 & \\
\hline
\end{tabular}

Tabla 4. Bahía Final. Cantidad y porcentaje de artefactos con diferentes porcentajes de corteza y estado, divididos por materia prima. E: enteros. Fg: fragmentados.

Bahía Final. Quantity and percentage of artifacts with different percentages of cortex and condition, divided by raw material. E: unbroken. Fg: broken.

\begin{tabular}{|c|c|c|c|c|c|c|c|}
\hline \multirow{2}{*}{ Bloque Antiguo } & \multicolumn{5}{|c|}{ Porcentaje De Corteza } & \multicolumn{2}{|c|}{ Estado } \\
\hline & 0 & 25 & 50 & 75 & 100 & $\mathrm{E}$ & $\mathrm{Fg}$ \\
\hline Volcanitas intermedias-básicas & 52 & 10 & 8 & 8 & 5 & 40 & 43 \\
\hline Sílex & 29 & 14 & 7 & 4 & 9 & 28 & 35 \\
\hline Calcedonia & 24 & 7 & 3 & 1 & 1 & 12 & 24 \\
\hline Jaspe & 1 & 4 & 1 & 1 & 1 & 5 & 3 \\
\hline Total porcentaje de corteza y estado (n) & 106 & 35 & 19 & 14 & 16 & 85 & 105 \\
\hline Total porcentaje de corteza y estado $(\%)$ & 55,79 & 18,42 & 10 & 7,37 & 8,42 & 44,74 & 55,26 \\
\hline \multicolumn{8}{|l|}{ Bloque Reciente } \\
\hline Sílex & 53 & 3 & 5 & 6 & 21 & 53 & 35 \\
\hline Calcedonia & 38 & 7 & 5 & 3 & 6 & 36 & 23 \\
\hline Volcanitas intermedias-básicas & 27 & 1 & 7 & 1 & 11 & 32 & 15 \\
\hline Volcanitas ácidas & 4 & 0 & 0 & 0 & 3 & 6 & 1 \\
\hline Total porcentaje de corteza y estado (n) & 122 & 11 & 17 & 10 & 41 & 127 & 74 \\
\hline Total porcentaje de corteza y estado $(\%)$ & 60,7 & 5,47 & 8,46 & 4,98 & 20,40 & 63,18 & 36,82 \\
\hline
\end{tabular}

que enteros, en el reciente esto se invierte. En este caso, el análisis de $\mathrm{X}^{2}$ dio como resultado diferencias estadísticamente significativas $\left(X^{2}=13,3, p\right.$-valor $\left.<0,01\right)$.

\section{Bajo de la Quinta}

Esta localidad arqueológica es una de las más importantes de la costa norte del golfo. Acá se recuperó un total de 1.823 artefactos, distribuidos como se muestra en la Tabla 5.
Nuevamente predominan los desechos por sobre las otras clases artefactuales, aunque existen diferencias entre los dos bloques. Mientras que en el antiguo los instrumentos se ubican en tercer lugar, en el reciente lo hacen en segundo. Además, en el caso de esta muestra es de destacar que los artefactos recuperados en el bloque reciente son más numerosos que los del bloque previo. Respecto de las materias primas, lo único que cambia es el segundo y tercer lugar en orden de importancia, que se intercambia entre la calcedonia (más usada 
Tabla 5. Bajo de la Quinta. Cantidad de artefactos divididos por las rocas más utilizadas.

Bajo de la Quinta. Lithic artifacts divided by most used rocks.

\begin{tabular}{lccccc}
\hline \multirow{2}{*}{ Bloque Antiguo } & \multicolumn{3}{c}{ Clase Artefactual } & Subtotal & Subtotal \\
& Lascas & Núcleos & Instrumentos & MP (n) & MP (\%) \\
\hline Sílex & 347 & 26 & 18 & 391 & 50,26 \\
\hline Volcanitas intermedias-básicas & 167 & 14 & 8 & 189 & 24,29 \\
\hline Calcedonia & 122 & 5 & 4 & 131 & 16,84 \\
\hline Volcanitas ácidas & 54 & 10 & 3 & 67 & 8,61 \\
\hline Subtotal clase de artefacto (n) & 690 & 55 & 33 & 778 & 100 \\
\hline Subtotal clase de artefacto (\%) & 88,69 & 7,07 & 4,24 & 100 & \\
\hline Bloque Reciente & & & & 641 & 61,34 \\
\hline Sílex & 579 & 24 & 38 & 242 & 23,16 \\
\hline Calcedonia & 228 & 5 & 9 & 127 & 12,15 \\
\hline Volcanitas intermedias-básicas & 121 & 3 & 3 & 35 & 3,35 \\
\hline Volcanitas ácidas & 31 & 1 & 3 & 1.045 & 100 \\
\hline Subtotal clase de artefacto (n) & 959 & 33 & 53 & 100 & \\
\hline Subtotal clase de artefacto (\%) & 91,77 & 3,16 & 5,07 & & \\
\hline
\end{tabular}

en el reciente) y las volcanitas intermedias-básicas, más usadas en el antiguo. El análisis de $\mathrm{X}^{2}$ arrojó diferencias estadísticamente significativas $\left(\mathrm{X}^{2}=78,37\right.$, p-valor $<0,01$ ).

De acuerdo con la Tabla 6, predominan los artefactos con $0 \%$ de reserva de corteza, aunque en el bloque antiguo siguen en importancia los de $100 \%$ y en el reciente los de $25 \%$. Las proporciones de enteros y fragmentados son similares en ambos bloques y las diferencias no son estadísticamente significativas $\left(\mathrm{X}^{2}=0,009, \mathrm{p}\right.$-valor $\left.>0,05\right)$.

\section{Caleta de los Loros}

En esta localidad arqueológica también se recuperó mayor cantidad de artefactos en el bloque reciente que en el tardío. Aquí predominan los desechos por sobre el resto de las categorías, aunque los porcentajes varían de bloque a bloque (Tabla 7). En el antiguo, núcleos e instrumentos aparecen en dimensiones iguales, mientras que en el reciente la proporción de núcleos es mayor que la de instrumentos.

En relación con las materias primas, en el bloque antiguo fueron usados principalmente el sílex y las rocas volcánicas ácidas, mientras que en el reciente predominan las rocas volcánicas intermedias-básicas y el sílex. La calcedonia se ubica siempre en último lugar. Estas diferencias son estadísticamente significativas $\left(\mathrm{X}^{2}=20,65\right.$, p-valor $\left.<0,01\right)$.

Los porcentajes de corteza contabilizados muestran que en ambos bloques hay mayormente artefactos con $0 \%$ de corteza en su cara dorsal, aunque son proporcionalmente más cantidad en el primero de ellos. El resto de los porcentajes son parejos en el bloque antiguo, mientras que en el tardío predominan los artefactos con $75 \%$ de corteza (Tabla 8). En ambos casos predominan artefactos fragmentados, aunque con diferencias que no son estadísticamente significativas entre bloques $\left(\mathrm{X}^{2}=0,11\right.$, p-valor $\left.>0,05\right)$.

\section{Bahía Creek}

En esta localidad se recuperó un total de 888 artefactos (Tabla 9). En esta muestra predominan los desechos tanto en el bloque antiguo como en el reciente, aunque la proporción es mucho mayor en el último, donde, además, baja la de núcleos e instrumentos. En relación con las materias primas, el uso es similar a lo largo de toda la ocupación, registrándose diferencias en el orden de importancia respecto del uso de las rocas volcánicas intermedias-básicas y la calcedonia. Además, el sílex es proporcionalmente más usado en los momentos recientes de la ocupación, mientras que el uso de las volcanitas intermedias-básicas cae sensiblemente para esos momentos. Estas diferencias son estadísticamente significativas $\left(\mathrm{X}^{2}=118,73\right.$, $\mathrm{p}$-valor $\left.<0,01\right)$.

Respecto del estado de los artefactos, predominan los enteros a lo largo de toda la ocupación, aunque la proporción de fragmentados aumenta en el bloque reciente hasta casi equiparar la de los enteros (Tabla 10). Estas diferencias son significativas $\left(X^{2}=16,7, p\right.$-valor $\left.<0,01\right)$. Los porcentajes de corteza se mantienen casi constantes en el tiempo.

\section{Bahía Rosas}

En esta última localidad se recuperaron 140 artefactos, divididos en partes iguales entre los dos bloques de ocupación. Cabe destacar que en el antiguo no se 
Tabla 6. Bajo de la Quinta. Cantidad y porcentaje de artefactos con diferentes porcentajes de corteza y estado, divididos por materia prima. E: enteros. Fg: fragmentados.

Bajo de la Quinta. Quantity and percentage of artifacts with different percentages of cortex and condition, divided by raw material. E: unbroken. Fg: broken.

\begin{tabular}{lccccccc}
\hline \multirow{2}{*}{ Bloque Antiguo } & \multicolumn{4}{c}{ Porcentaje de Corteza } & \multicolumn{3}{c}{ Estado } \\
\cline { 2 - 8 } & 0 & 25 & 50 & 75 & 100 & E & Fg \\
\hline Sílex & 273 & 31 & 30 & 24 & 33 & 211 & 180 \\
\hline Volcanitas intermedias-básicas & 128 & 16 & 17 & 12 & 16 & 101 & 88 \\
\hline Calcedonia & 103 & 5 & 11 & 5 & 7 & 59 & 72 \\
\hline Volcanitas ácidas & 41 & 2 & 3 & 11 & 10 & 41 & 26 \\
\hline$\quad$ Total porcentaje de corteza y estado (n) & 545 & 54 & 61 & 52 & 66 & 412 & 366 \\
\hline$\quad$ Total porcentaje de corteza y estado (\%) & 70,05 & 6,94 & 7,84 & 6,68 & 8,48 & 52,96 & 47,04 \\
\hline Bloque Reciente & & & & & & & \\
\hline Sílex & 442 & 69 & 42 & 29 & 59 & 351 & 290 \\
\hline Calcedonia & 191 & 18 & 9 & 8 & 16 & 114 & 128 \\
\hline Volcanitas intermedias-básicas & 92 & 8 & 10 & 4 & 13 & 68 & 59 \\
\hline Volcanitas ácidas & 24 & 4 & 3 & 1 & 3 & 18 & 17 \\
\hline$\quad$ Total porcentaje de corteza y estado (n) & 749 & 99 & 64 & 42 & 91 & 551 & 494 \\
\hline Total porcentaje de corteza y estado (\%) & 71,67 & 9,47 & 6,12 & 4,02 & 8,71 & 52,73 & 47,27 \\
\hline
\end{tabular}

Tabla 7. Caleta de los Loros. Cantidad de artefactos divididos por las rocas más utilizadas.

Caleta de los Loros. Lithic artifacts divided by most used rocks.

\begin{tabular}{|c|c|c|c|c|c|}
\hline \multirow{2}{*}{ Bloque Antiguo } & \multicolumn{3}{|c|}{ Clase Artefactual } & \multirow{2}{*}{$\begin{array}{l}\text { Subtotal } \\
\text { MP (n) }\end{array}$} & \multirow{2}{*}{$\begin{array}{l}\text { Subtotal } \\
\text { MP }(\%)\end{array}$} \\
\hline & Lascas & Núcleos & Instrumentos & & \\
\hline Sílex & 23 & 1 & 1 & 25 & 54,35 \\
\hline Volcanitas ácidas & 10 & 0 & 0 & 10 & 21,74 \\
\hline Volcanitas intermedias-básicas & 6 & 1 & 1 & 8 & 17,39 \\
\hline Calcedonia & 3 & 0 & 0 & 3 & 6.52 \\
\hline Subtotal clase de artefacto (n) & 42 & 2 & 2 & 46 & 100 \\
\hline Subtotal clase de artefacto $(\%)$ & 91,3 & 4,35 & 4,35 & 100 & \\
\hline \multicolumn{6}{|l|}{ Bloque Reciente } \\
\hline Volcanitas intermedias-básicas & 33 & 10 & 3 & 46 & 57,5 \\
\hline Sílex & 22 & 1 & 0 & 23 & 28,75 \\
\hline Volcanitas ácidas & 6 & 0 & 0 & 6 & 7,5 \\
\hline Calcedonia & 3 & 1 & 1 & 5 & 6,25 \\
\hline Subtotal clase de artefacto (n) & 64 & 12 & 4 & 80 & 100 \\
\hline Subtotal clase de artefacto (\%) & 80 & 15 & 5 & 100 & \\
\hline
\end{tabular}

registraron instrumentos, mientras que en el reciente la proporción de esta categoría supera a la de los núcleos (Tabla 11). Los desechos son mayoritarios a lo largo de toda la ocupación.

En relación con las materias primas, el uso es predominantemente local (volcanitas), registrándose gran diferencia con la roca que sigue (sílex). Calcedonia y volcanitas ácidas invierten su orden de importancia para momentos tardíos. Estas diferencias no son estadísticamente significativas $\left(X^{2}=3,96\right.$, p-valor $>0,05$ ).
Dentro del estado de los artefactos, se observó que los porcentajes entre enteros y fragmentados son muy similares en ambos bloques, aunque se invierten para momentos tardíos (Tabla 12). Los resultados del análisis de $\mathrm{X}^{2}$ arrojaron resultados no estadísticamente significativos $\left(\mathrm{X}^{2}=0,11 \mathrm{p}\right.$-valor $\left.>0,05\right)$. Los porcentajes de corteza también varían: mientras que en el bloque antiguo predominan aquellos con $0 \%$ en su cara dorsal, seguidos de los de $100 \%$, en el reciente predominan también los de $0 \%$ pero siguen en orden los de $25 \%$, mientras que los de $100 \%$ se ubican en cuarto lugar. 
Tabla 8. Caleta de los Loros. Cantidad y porcentaje de artefactos con diferentes porcentajes de corteza y estado, divididos por materia prima. E: enteros. Fg: fragmentados.

Caleta de los Loros. Quantity and percentage of artifacts with different percentages of cortex and condition, divided by raw material. E: unbroken. Fg: broken.

\begin{tabular}{lccccccc}
\hline \multirow{2}{*}{ Bloque Antiguo } & \multicolumn{3}{c}{ Porcentaje de Corteza } & \multicolumn{3}{c}{ Estado } \\
& 0 & 25 & 50 & 75 & 100 & E & Fg \\
\hline Sílex & 20 & 1 & 1 & 2 & 1 & 12 & 13 \\
\hline Volcanitas ácidas & 8 & 1 & 0 & 0 & 1 & 4 & 6 \\
\hline Volcanitas intermedias-básicas & 6 & 1 & 1 & 0 & 0 & 4 & 4 \\
\hline Calcedonia & 2 & 0 & 0 & 1 & 0 & 1 & 2 \\
\hline Total porcentaje de corteza y estado (n) & 36 & 3 & 2 & 3 & 2 & 21 & 25 \\
\hline Total porcentaje de corteza y estado (\%) & 78,26 & 6,52 & 4,35 & 6,52 & 4,35 & 45,65 & 54,35 \\
\hline Bloque Reciente & & & & & & & \\
\hline Volcanitas intermedias-básicas & 19 & 6 & 4 & 15 & 2 & 23 & 23 \\
\hline Sílex & 17 & 2 & 2 & 1 & 1 & 9 & 14 \\
\hline Volcanitas ácidas & 4 & 1 & 0 & 1 & 0 & 1 & 5 \\
\hline Calcedonia & 4 & 0 & 0 & 1 & 0 & 1 & 4 \\
\hline Total porcentaje de corteza y estado (n) & 44 & 9 & 6 & 18 & 3 & 34 & 46 \\
\hline Total porcentaje de corteza y estado (\%) & 55,00 & 11,25 & 7,5 & 22,5 & 3,75 & 42.5 & 57,5 \\
\hline
\end{tabular}

Tabla 9. Bahía Creek. Cantidad de artefactos divididos por las rocas más utilizadas.

Bahía Creek. Lithic artifacts divided by most used rocks.

\begin{tabular}{lccccc}
\hline \multirow{2}{*}{ Bloque Antiguo } & \multicolumn{3}{c}{ Clase Artefactual } & Subtotal & Subtotal \\
& Lascas & Núcleos & Instrumentos & MP (N) & MP (\%) \\
\cline { 2 - 6 } Sílex & 149 & 37 & 5 & 191 & 38,2 \\
\hline Volcanitas intermedias-básicas & 142 & 30 & 5 & 177 & 35,4 \\
\hline Calcedonia & 70 & 21 & 4 & 95 & 19 \\
\hline Volcanitas ácidas & 22 & 12 & 3 & 37 & 7,4 \\
\hline \multicolumn{1}{c}{ Subtotal clase de artefacto (n) } & 383 & 100 & 17 & 500 & 100 \\
\hline Subtotal clase de artefacto (\%) & 76,6 & 20 & 3,4 & 100 & \\
\hline Bloque Reciente & & & & 250 & 64,43 \\
\hline Sílex & 223 & 23 & 4 & 100 & 25,77 \\
\hline Calcedonia & 95 & 3 & 2 & 32 & 8,25 \\
\hline Volcanitas intermedias-básicas & 29 & 3 & 0 & 6 & 1,55 \\
\hline Volcanitas ácidas & 6 & 0 & 0 & 388 & 100 \\
\hline Subtotal clase de artefacto (n) & 353 & 29 & 6 & 100 & \\
\hline Subtotal clase de artefacto (\%) & 90,98 & 7,47 & 1,55 & & 25 \\
\hline
\end{tabular}


Tabla 10. Bahía Creek. Cantidad y porcentaje de artefactos con diferentes porcentajes de cortezay estado, divididos pormateria prima. E: enteros. Fg: fragmentados. Bahía Creek. Quantity and percentage of artifacts with different percentages of cortex and condition, divided by raw material. E: unbroken. Fg: broken.

\begin{tabular}{|c|c|c|c|c|c|c|c|}
\hline \multirow{2}{*}{ Bloque Antiguo } & \multicolumn{5}{|c|}{ Porcentaje de Corteza } & \multicolumn{2}{|c|}{ Estado } \\
\hline & 0 & 25 & 50 & 75 & 100 & E & $\mathrm{Fg}$ \\
\hline Sílex & 94 & 22 & 31 & 20 & 24 & 131 & 60 \\
\hline Volcanitas intermedias-básicas & 103 & 24 & 24 & 11 & 15 & 105 & 72 \\
\hline Calcedonia & 52 & 23 & 9 & 7 & 4 & 55 & 40 \\
\hline Volcanitas ácidas & 6 & 3 & 7 & 10 & 11 & 31 & 6 \\
\hline Total porcentaje de corteza y estado (n) & 255 & 72 & 71 & 48 & 54 & 322 & 178 \\
\hline Total porcentaje de corteza y estado (\%) & 51 & 14,4 & 14,2 & 9,6 & 10,8 & 64,4 & 35,6 \\
\hline \multicolumn{8}{|l|}{ Bloque Reciente } \\
\hline Sílex & 117 & 40 & 32 & 35 & 26 & 123 & 127 \\
\hline Calcedonia & 56 & 15 & 15 & 6 & 8 & 51 & 49 \\
\hline Volcanitas intermedias-básicas & 17 & 3 & 4 & 3 & 5 & 21 & 11 \\
\hline Volcanitas ácidas & 4 & 0 & 1 & 0 & 1 & 2 & 4 \\
\hline Total porcentaje de corteza y estado (n) & 194 & 58 & 52 & 44 & 40 & 197 & 191 \\
\hline Total porcentaje de corteza y estado $(\%)$ & 50 & 14,95 & 13,4 & 11,34 & 10,31 & 50,77 & 49,23 \\
\hline
\end{tabular}

Tabla 11. Bahía Rosas. Cantidad de artefactos divididos por las rocas más utilizadas.

Bahía Rosas. Lithic artifacts divided by most used rocks.

\begin{tabular}{|c|c|c|c|c|c|}
\hline \multirow{2}{*}{ Bloque Antiguo } & \multicolumn{3}{|c|}{ Clase Artefactual } & \multirow{2}{*}{$\begin{array}{l}\text { Subtotal } \\
\text { MP (n) }\end{array}$} & \multirow{2}{*}{$\begin{array}{c}\text { Subtotal MP } \\
(\%)\end{array}$} \\
\hline & Lascas & Núcleos & Instrumentos & & \\
\hline Volcanitas intermedias-básicas & 41 & 10 & 0 & 51 & 72,86 \\
\hline Sílex & 11 & 2 & 0 & 13 & 18,57 \\
\hline Volcanitas ácidas & 5 & 0 & 0 & 5 & 7,14 \\
\hline Calcedonia & 1 & 0 & 0 & 1 & 1,43 \\
\hline Subtotal clase de artefacto (n) & 58 & 12 & 0 & 70 & 100 \\
\hline Subtotal clase de artefacto (\%) & 82,86 & 17,14 & 0 & 100 & \\
\hline \multicolumn{6}{|l|}{ Bloque Reciente } \\
\hline Volcanitas intermedias-básicas & 38 & 3 & 9 & 50 & 71,43 \\
\hline Sílex & 8 & 1 & 4 & 13 & 18,57 \\
\hline Calcedonia & 5 & 0 & 0 & 5 & 7,14 \\
\hline Volcanitas ácidas & 2 & 0 & 0 & 2 & 2,86 \\
\hline Subtotal clase de artefacto (n) & 53 & 4 & 13 & 70 & 100 \\
\hline Subtotal clase de artefacto $(\%)$ & 75,71 & 5,71 & 18,57 & 100 & \\
\hline
\end{tabular}

Tabla 12. Bahía Rosas. Cantidad y porcentaje de artefactos con diferentes porcentajes de corteza y estado, divididos por materia prima. E: enteros. Fg: fragmentados.

Bahía Rosas. Quantity and percentage of artifacts with different percentages of cortex and condition, divided by raw material. E: unbroken. Fg: broken.

\begin{tabular}{|c|c|c|c|c|c|c|c|}
\hline \multirow{2}{*}{ Bloque Antiguo } & \multicolumn{5}{|c|}{ Porcentaje de Corteza } & \multicolumn{2}{|c|}{ Estado } \\
\hline & 0 & 25 & 50 & 75 & 100 & E & $\mathrm{Fg}$ \\
\hline Volcanitas intermedias-básicas & 17 & 11 & 7 & 3 & 13 & 27 & 24 \\
\hline Sílex & 3 & 0 & 1 & 4 & 5 & 5 & 8 \\
\hline Volcanitas ácidas & 2 & 1 & 1 & 0 & 1 & 3 & 2 \\
\hline Calcedonia & 1 & 0 & 0 & 0 & 0 & 1 & 0 \\
\hline Total porcentaje de corteza y estado (n) & 23 & 12 & 9 & 7 & 19 & 36 & 34 \\
\hline Total porcentaje de corteza y estado (\%) & 32,86 & 17,14 & 12,86 & 10 & 27,14 & 51,43 & 48,57 \\
\hline \multicolumn{8}{|l|}{ Bloque Reciente } \\
\hline Volcanitas intermedias-básicas & 19 & 11 & 10 & 4 & 6 & 24 & 26 \\
\hline Sílex & 6 & 3 & 0 & 3 & 1 & 8 & 5 \\
\hline Calcedonia & 1 & 1 & 0 & 1 & 2 & 1 & 4 \\
\hline Volcanitas ácidas & 2 & 0 & 0 & 0 & 0 & 1 & 1 \\
\hline Total porcentaje de corteza y estado (n) & 28 & 15 & 10 & 8 & 9 & 34 & 36 \\
\hline Total porcentaje de corteza y estado (\%) & 40 & 21,43 & 14,29 & 11,43 & 12,86 & 48,57 & 51,43 \\
\hline
\end{tabular}




\section{Análisis estadísticos}

A partir de la realización del análisis multifactorial, se comprobó que el 46,2\% de la variación de la muestra total está comprendida en el primer eje (horizontal, Dimensión 1) de la Figura 2. Tal como se observa en el gráfico, a lo largo de la dimensión horizontal se agrupan diferentes sitios que serían más similares entre sí, considerando ambos bloques temporales con sus distintas variables. De esta manera, por ejemplo ambos bloques temporales del sitio BQ son parecidos entre sí, mientras que los de la localidad BC son muy diferentes, ya que se encuentran alejados sobre el eje horizontal. Los casos que poseen frecuencias más elevadas de las variables analizadas son aquellos que se encuentran hacia la derecha del eje horizontal. El eje vertical, por su parte, explica el 26,8\% de la variabilidad observada en el análisis (Figura 2). Por ejemplo, ambos momentos de ocupación de SAO están asociados entre sí. En cambio, las ocupaciones de BF están claramente separadas, por lo que serían muy diferentes en su composición. En el caso de

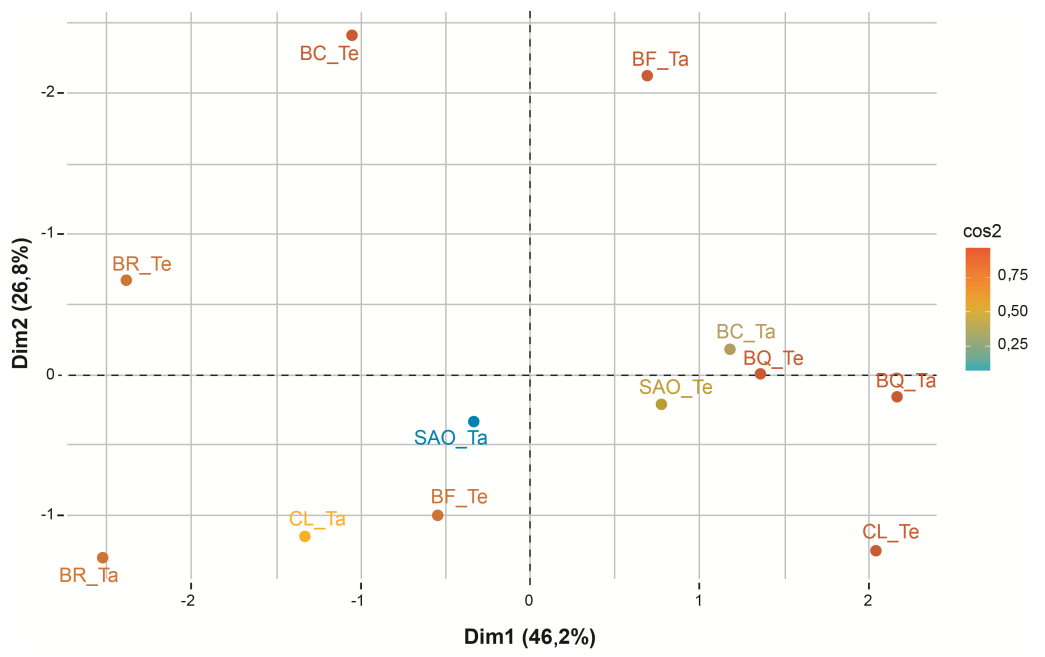

Figura 2. Análisis factorial múltiple. Se ubican las localidades en relación a las dos dimensiones del análisis (1 y 2). Las localidades cuyas siglas están en naranja oscuro son las que más aportan a la variación. Multiple factorial analysis. Archaeological localities are placed in relation to the two dimensions of the analysis ( 1 and 2). The dark orange dots are the localities with greater contribution to the variation.

este eje, las localidades que presentan valores más altos de las variables analizadas son las que se encuentran de la mitad hacia arriba.

En la Figura 3 se muestran las variables más importantes en el análisis para ambos ejes. En la Figura 3.A son la materia prima, la clase de artefactos y el porcentaje de corteza en el caso del eje horizontal. En la Figura 3.B se muestra que, dentro de estos grupos de variables, las que más inciden son el porcentaje 0 de corteza, la proporción de lascas y núcleos y el uso de las volcanitas intermedias-básicas y el sílex. De esta manera, por ejemplo, se podría afirmar que la ocupación tardía de $\mathrm{BC}$ posee mayor proporción de estos indicadores que su contraparte del bloque temprano. En cambio, en el caso de BQ, dichas proporciones no variaron significativamente.

En el caso del eje vertical, tal como se muestra en la Figura 3.C, el estado y la clase de artefactos son las variables que más inciden en las diferencias detectadas entre las ocupaciones de las localidades. Al discriminar estos dos indicadores se observa que los artefactos enteros son los que introducen la mayor variabilidad, junto con los núcleos y los instrumentos (Figura 3.D). En este sentido, por ejemplo, las ocupaciones tardías de BF tendrían más artefactos enteros que las ocupaciones tempranas, pero proporciones similares de los mismos en relación con las ocupaciones tempranas de BC.

Por último, se presenta en la Figura 4 la asociación de variables. Se puede observar en el gráfico que clase, corteza y materia prima se encuentran relacionadas entre sí sobre la primera dimensión, es decir, los conjuntos que tienden a agruparse sobre este eje poseen proporciones similares de estas categorías. La variable estado se diferencia claramente de las otras tres en ambos ejes, actuando de manera independiente. Finalmente, la localización, que fue tomada como una variable pasiva, no introduce diferencias relevantes; es decir, la composición de los conjuntos y la variación de los mismos no dependen de su localización en el espacio. 

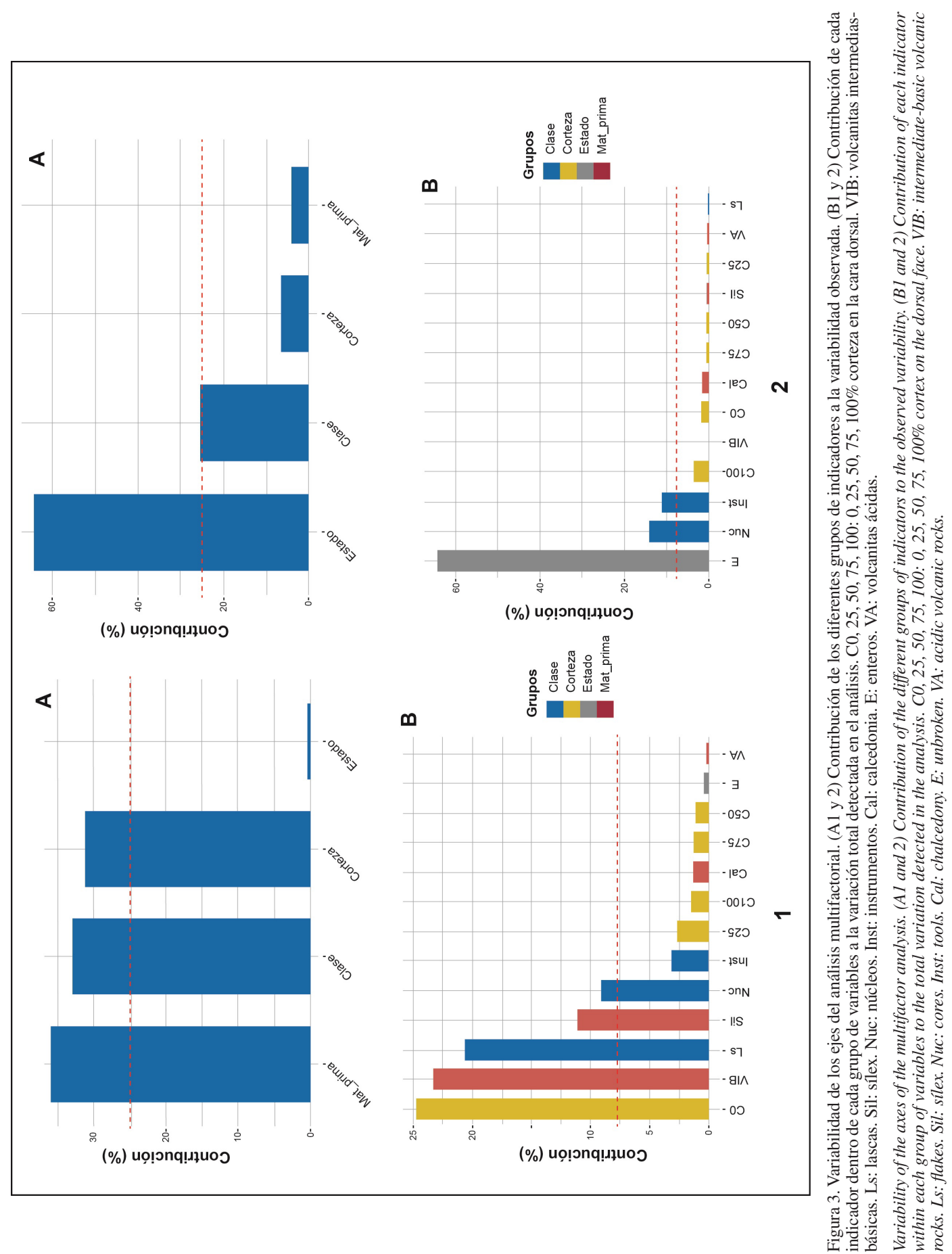


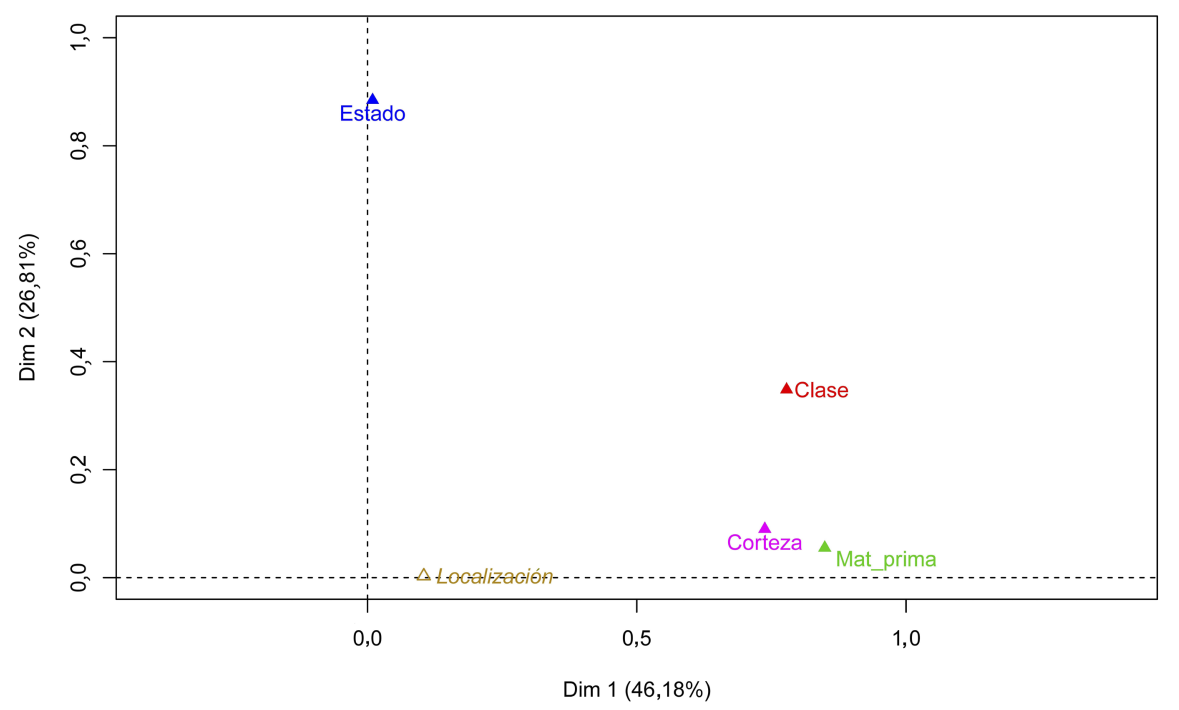

Figura 4. Contribución de cada grupo de variables al análisis multifactorial.

Contribution of each group of variables to the multiple factor analysis.

\section{Discusión y Conclusiones}

Los análisis realizados permitieron delinear ciertas tendencias en las localidades estudiadas. En primer lugar, se pudo establecer que el uso de las rocas varió a lo largo del tiempo, siendo estas diferencias significativas en cinco de las seis localidades estudiadas. El caso excepcional lo constituye BR, la localidad ubicada más hacia el este de la costa y en donde no hay diferencias entre bloques temporales. Allí, el uso local de rocas se mantuvo a lo largo del tiempo; en cambio, en el resto de las localidades se usaron principalmente rocas locales en momentos tempranos de la ocupación, y extra areales en momentos tardíos. Esto puede deberse a la decisión de llevar hacia las localidades de la costa norte rocas de mejor calidad para la talla provenientes de otros sectores, como son sílices $\mathrm{y}$ calcedonias que en las fuentes de materia prima de esa región son de aparición muy escasa (Alberti 2016). Estas rocas habrían sido necesarias para la producción de instrumentos confiables que pudiesen ser usados en el marco de variadas actividades, tal como lo habría requerido el aumento del riesgo planteado para los momentos tardíos de la ocupación del golfo (Cardillo y Favier Dubois 2010). Dentro de las localidades en las que las diferencias en el uso de las materias primas son significativas, CL es el único caso en el cual las rocas volcánicas intermedias-básicas ocuparon un lugar más importante para momentos tardíos, lo cual indica que el uso de rocas fue siempre principalmente local. Esto podría deberse a que la mencionada localidad registra ocupaciones efímeras y no redundantes o extendidas en el tiempo, con lo cual el uso de las rocas locales habría sido suficiente para la manufactura de herramientas para las actividades que se habrían llevado a cabo en este lugar, es decir, no se habrían necesitado materias primas de alta calidad para la talla.

En relación con los tipos de artefactos de los conjuntos, predominan siempre los desechos. Este hecho se verifica, en general, en todas las localidades arqueológicas de la costa rionegrina. El segundo lugar está ocupado por los núcleos en el bloque temprano de todas las localidades, mientras que en el bloque tardío este lugar es ocupado por los instrumentos solamente en BR y BQ. En el resto de las localidades siguen siendo los núcleos la clase artefactual de segundo orden de importancia. El alto descarte de núcleos puede obedecer a la abundancia de materia prima registrada, que implicaría el inicio de nuevas secuencias de talla cada vez que fuese necesario. En dichos sitios, entonces, se podrían haber manufacturado instrumentos que luego eran transportados hacia otros lugares. En cambio, en BR y BQ se habría practicado un equipamiento del espacio (sensu Kuhn 2004) por diferentes motivos. En la primera localidad esto podría haberse dado por la relativa escasez de materia prima de excelente calidad en las cercanías, y en la segunda por ser una localidad que presenta evidencias de una alta intensidad de ocupación a lo largo del tiempo: la reocupación del lugar acarrearía la necesidad de contar en el lugar con herramientas para la explotación de los recursos.

$\mathrm{Al}$ analizar los porcentajes de corteza presentes en la cara dorsal de los artefactos, se puede observar que el $0 \%$ de corteza ocupa siempre el primer lugar. 
Esto indicaría que invariablemente las tareas iniciales de descortezamiento de nódulos y primeros estadios de manufactura de los artefactos no se habrían llevado a cabo en estos lugares. Esto es de destacar: debido a que las fuentes de materia prima están disponibles a lo largo de toda el área, sería esperable el transporte de las mismas hacia los lugares de habitación. Esto es particularmente aplicable a las rocas locales, no así a los sílices y la calcedonia que vendrían de lugares más alejados de la costa rionegrina, o de sitios hacia el interior del continente. Sin embargo, el patrón de porcentaje de corteza se mantiene, independientemente del tipo de roca considerado. Esto podría deberse a que en las fuentes no todas las rocas son aptas para la talla, por lo cual, a pesar de su amplia disponibilidad, se podrían realizar en ellas tareas de testeo de nódulos para llevar hacia los lugares de habitación únicamente aquellos idóneos para la manufactura de artefactos.

Finalmente, respecto del porcentaje de artefactos enteros y fragmentados, hay situaciones variadas. En localidades como SAO, BQ, CL y BR las diferencias en las proporciones de artefactos enteros y fragmentados a lo largo del tiempo no son estadísticamente significativas y estarían relacionadas con el azar. SAO y BQ son localidades que han sido recurrentemente ocupadas en el tiempo, por lo que en estos lugares se pueden haber dado actividades de recambio de artefactos y de equipamiento del espacio indistintamente a lo largo de los años, lo que atenuaría las diferencias entre las proporciones de artefactos enteros y fragmentados. En cambio, en BF y BC existen diferencias significativas que señalan que, en el primer caso, la proporción de artefactos enteros aumenta para momentos tardíos, dándose el caso inverso en $\mathrm{BC}$, y este fenómeno no está relacionado con el azar. En el caso de BC puede deberse a la intensa perturbación a la que localidad se ha visto sometida a lo largo de los años (para más detalle ver Carranza 2015). En el caso de BF, los sitios no han sufrido gran impacto como consecuencia del desarrollo de actividades antrópicas (por ejemplo, la circulación de cuatriciclos), por lo que se podría pensar que los conjuntos presentan una integridad y una resolución mayores a lo que ocurre en otros lugares de la costa.

En relación con los resultados del análisis multifactorial, observamos que existen localidades en las que ambas ocupaciones se encuentran relacionadas entre sí, demostrando similitud a lo largo del tiempo en las variables analizadas. Es el caso de BQ y SAO. En el primer caso, tomando en cuenta el eje horizontal del análisis, $\mathrm{BQ}$ posee proporciones similares a lo largo del tiempo en lo que respecta a las materias primas, los tipos de artefactos presentes en el sitio y el porcentaje de corteza que predomina en la muestra, lo que daría cuenta de actividades similares de extracción y procesamiento de recursos realizadas a lo largo de toda la ocupación. En el segundo caso, aunque ambas ocupaciones son similares, en el bloque temprano de $\mathrm{SAO}$ las proporciones de las variables antes mencionadas son mayores que en el tardío. En esta localidad los cambios en la línea de costa durante el Holoceno (Favier Dubois y Scartascini 2012), podrían haber llevado a un cambio en las actividades realizadas en el lugar a lo largo del tiempo, lo cual se reflejaría en estas diferencias en las variables analizadas.

El resto de las localidades analizadas son muy diferentes entre sí. La mayor proporción de las variables analizadas en el eje horizontal se encuentra en el bloque tardío de BF y BC, y en el temprano de SAO y CL. Por su parte, los dos bloques de ocupación de BR son distintos entre sí, pero no poseen proporciones altas de ninguna de las variables consideradas. Entonces, se observa que mayor proporción de volcanitas intermedias-básicas y sílex, lascas y núcleos y porcentajes 0 de corteza se encuentran en las ocupaciones tardías de BF y BC, y en las tempranas de $\mathrm{SAO}$ y $\mathrm{CL}$. En estas dos últimas, para momentos tardíos habría disminuido el uso de las mencionadas rocas, de la aparición de lascas y núcleos y de los porcentajes 0 de corteza, a la inversa de BF y BC.

En trabajos previos (Alberti 2016) se realizaron análisis acerca de la explotación y uso de las materias primas líticas a lo largo de toda la costa rionegrina, comparándose entre sí ambas zonas de la costa a escala regional. Los resultados alcanzados indican que las estrategias de reducción de rocas se mantuvieron constantes a lo largo de toda la ocupación del litoral rionegrino, y que los cambios en el nivel del mar no trajeron aparejados cambios en los tipos de rocas explotados (Alberti 2016). Para la costa norte se propuso un uso predominantemente no local de rocas durante toda la ocupación, pese a la reducción de la movilidad propuesta para momentos tardíos (Favier Dubois et al. 2009). Sin embargo, al cambiar la escala de análisis y discriminar por localidades, este panorama cambia: el uso preponderante de rocas no locales se verifica solamente en los momentos tardíos de la ocupación. En este sentido, entonces, en las localidades tomadas en este trabajo el aumento del riesgo registrado para momentos tardíos, con la consecuente ampliación de la dieta (Cardillo y Favier Dubois 2010; Favier Dubois et al. 2009), habría implicado que los grupos prefiriesen las rocas no locales debido a su mayor calidad para la talla. Estas materias primas habrían permitido la disminución de fallas durante el proceso de manufactura de instrumentos más confiables, necesarios para enfrentarse a contextos de reducción de la movilidad, restricción del espacio y ampliación de la variabilidad de recursos consumidos.

En este trabajo se presentaron los resultados del análisis exploratorio de un grupo de variables aplicado a seis localidades de la costa norte del golfo San Matías. 
Los mismos indican una diferencia sustancial respecto de lo observado previamente en el panorama regional, y gran variabilidad a lo largo del tiempo relacionada con, principalmente, las materias primas explotadas. De esta forma, este artículo se constituye en una nueva arista para explorar las tendencias en la tecnología lítica de los grupos cazadores-recolectores-pescadores que ocuparon la costa rionegrina durante el Holoceno Medio y Tardío.
Agradecimientos: AlDr. Marcelo Cardillo por la ayuda brindada para la realización de los análisis estadísticos. $\mathrm{Al}$ equipo de investigación por la colaboración en las tareas de campo. Los datos presentados en este trabajo provienen de las tareas de análisis realizadas durante la beca doctoral de la autora, otorgada por el CONICET. A los revisores anónimos cuyos comentarios y sugerencias mejoraron el trabajo original. Al Comité Editorial de la revista por la labor realizada.

\section{Referencias Citadas}

Alberti, J. 2012. Fuentes de rocas y uso de materias primas líticas en Bahía Final 6, costa norte del golfo San Matías (Río Negro, Argentina). Intersecciones en Antropología 13:237-249.

Alberti, J. 2016. Disponibilidad y Explotación de Materias Primas Líticas en la Costa de Norpatagonia (Argentina). Un Enfoque Regional, British Archaeological Reports, International Series 1901, Archaeopress, Oxford.

Alberti, J. y M. Cardillo 2015. Primary and secondary lithic raw material sources along the western coast of San Matías Gulf (Río Negro province, Argentina): A first approach to their spatial variability. Quaternary International 373:63-71.

Alberti, J., M. Cardillo y C. Favier Dubois 2015. Fuentes de materias primas líticas en la costa del golfo San Matías (provincia de Río Negro, Argentina). Una síntesis regional. En Intersecciones en Antropología, Volumen Especial 2 "Materias primas líticas en Patagonia. Localización, circulación y métodos de estudio de las fuentes de rocas de la Patagonia argentino-chilena", editado por J. Alberti y V. Fernández, pp. 27-37.

Alberti, J.y V.Fernández 2015. Propuestaclasificatoria para las materias primas líticas en Patagonia (Argentina). Arqueología 21 (2):211-235.

Andrefsky, W. 1994. Raw-material availability and the organization of technology. American Antiquity 59 (1):21-34.

Aragón, E. y N. Franco 1997. Características de rocas para la talla por percusión y propiedades petrográficas. Anales del Instituto de la Patagonia 25:187-189.

Aschero, C. 1975. Ensayo para una clasificación morfológica de artefactos líticos aplicada a estudios tipológicos comparativos. Manuscrito presentado al CONICET, Buenos Aires.

Aschero, C. 1983. Ensayo para una clasificación morfológica de artefactos líticos. Revisión del año 1975. Manuscrito presentado al CONICET, Buenos Aires.

Beck, C., A. Taylor, G. Jones, C. Fadem, C. Cook y S. Millward 2002. Rocks are heavy: Transport costs and Paleoarchaic quarry behavior in the Great Basin. Journal of Anthropological Archaeology 21:481-507.

Borcard, D., F. Gillet y P. Legendre 2011. Numerical ecology with R. Journal of Agricultural, Biological, and Environmental Statistics 17 (2):308-309.

Cardillo, M. y C. Favier Dubois 2010. Una aproximación al uso del espacio en la Costa Norte del Golfo San Matías (Río Negro, Argentina): relaciones entre la evidencia artefactual e isotópica. En III Jornadas Interdisciplinarias de Historia y Ciencias Humanas. Movilidad y Migraciones, editado por A. Guiance, pp. 241-252. IMHICIHUCONICET, Buenos Aires.

Carranza, E. 2015. Análisis de Conjuntos Líticos de Superficie desde una Perspectiva Tafonómica. Estudios en la Costa Norte del Golfo
San Matías, Río Negro, Argentina. Tesis de Licenciatura, Facultad de Filosofía y Letras, Universidad de Buenos Aires, Buenos Aires.

Civalero, T. y N. Franco 2003. Early human occupations in Western Santa Cruz Province, Southermost South America. Quaternary International 109-110:77-86.

Codignotto, J., C. Beros y L. Trebino 1987. Nuevo método cronoestratigráfico, morfocronología en secuencia deposicional cordoniforme. Revista de la Asociación Geológica Argentina $42(3-4): 462-468$.

Ericson, J. 1984. Toward the analysis of lithic reduction systems. En Prehistoric Quarries and Lithic Production, editado por J. Ericson y B. Purdy, pp. 1-19. Cambridge University Press, Cambridge.

Escoffier, B. y J. Pagès 1998. Analyse Factorielles Simples Et Multiples: Objectifs, Méthodes Et Interprétation. Dunod, París.

Etcheverría, M., A. Folguera, C. Dal Molín, M. Dalponte y G. Ferro 2006. Hojas Geológicas 4163-II/IV y I/III, Viedma y General Conesa. Provincias de Río Negro y Buenos Aires. Instituto de Geología y Recursos Minerales, Servicio Geológico Minero Argentino (SEGEMAR), Boletín 366. Buenos Aires.

Favier Dubois, C. 2013. Hacia una cronología del uso del espacio en la costa norte del golfo San Matías (Río Negro, Argentina): sesgos geológicos e indicadores temporales. En Tendencias TeóricoMetodológicas y Casos de Estudio en la Arqueología de Patagonia, editado por F. Zangrando, R. Barberena, A. Gil, G. Neme, M. Giardina, L. Luna, C. Otaola, S. Paulides, L. Salgán y A. Tívoli, pp. 87-96. Museo de Historia Natural de San Rafael, San Rafael.

Favier Dubois, C. y J.Alberti 2014. Materias primas líticas en la costa norte del golfo San Matías (Río Negro, Argentina): distribución de fuentes y tendencias generales en su aprovechamiento. Revista del Museo de Antropología 7 (1):93-104.

Favier Dubois, C., F. Borella y R. Tykot 2009. Explorando tendencias en el uso humano del espacio y los recursos en el litoral rionegrino (Argentina) durante el Holoceno medio y tardío. En Arqueología de la Patagonia - Una Mirada desde el Último Confín, editado por M. Salemme, F. Santiago, M. Álvarez, E. Piana, M. Vázquez y E. Mansur, pp. 985-997. Editorial Utopías, Ushuaia.

Favier Doubois, C. y R. Kokot 2011. Changing scenarios in Bajo de la Quinta (San Matías Gulf, Northern Patagonia, Argentina): Impact of geomorphologic processes in subsistence and human use of coastal habitats. Quaternary International 245:103-110.

Favier Dubois, C., R. Kokot, F. Scartascini y F. Borella 2016. Una perspectiva geoarqueológica del registro de ocupaciones humanas en el Golfo San Matías (Río Negro, Argentina). Intersecciones en Antropología, "La geoarqueología en Argentina: aportes y avances", Volumen Especial 4:47-59. 
Favier Dubois, C. y F. Scartascini 2012. Intensive fishery scenarios on the North Patagonian coast (Río Negro, Argentina) during the Mid-Holocene. Quaternary International 256:62-70.

Franco, N. 2002. Estrategias de Utilización de Recursos Líticos en la Cuenca Superior del Río Santa Cruz (Argentina). Tesis Doctoral, Facultad de Filosofía y Letras, Universidad de Buenos Aires, Buenos Aires.

Franco, N. y L. Borrero 1999. Metodología de análisis de la estructura regional de recursos líticos. En En los Tres Reinos. Prácticas de Recolección en el Cono Sur de América, editado por C. Aschero, A. Korstanje y P. Vuoto, pp. 27-37. Magua Publicaciones, San Miguel de Tucumán.

Freedman, D., R. Pisani, R. Purves y A. Adhikari 1993. Estadística. Segunda edición. Antoni Bosch editor, Barcelona.

Gelós, E.M., J.O. Spagnuolo y R.A. Schillizzi 1988. Las unidades morfológicas de la costa norte del golfo San Matías y su evolución. Revista de la Asociación Geológica Argentina 43 (3):315-326.

Gelós, E.M., J.O. Spagnuolo y R.A. Schillizzi 1990. Caracteres texturales y mineralógicos de sedimentos de playa de la costa norte del golfo San Matías. Revista de la Asociación Argentina de Mineralogía, Petrología y Sedimentología 21 (1/4):41-52.

González Díaz, E. y E. Malagnino 1984. Geomorfología de la provincia de Río Negro. Actas del IX Congreso Geológico Argentino. pp. 7-149, Gobierno de la Provincia de Río Negro, Secretaría de Recuersos Naturales, Viedma.

Hammer, Ø., D. Harper y P. Ryan 2001. PAST. Palaeontological Statistics software package for education and data analysis. Palaeontologia Electronica 4 (1):1-9.

Kuhn, S. 2004. Upper Paleolithic raw material economies at Ücagızlı cave, Turkey. Journal of Anthropological Archaeology 23:431-448.

Legendre, P. y L. Legendre 1998. Numerical Ecology. Elsevier, Amsterdam.
Martínez, H., C. Náñez, A. Lizuain, C. Dal Molín y A. Turel 2001. Hoja Geológica 4166-II, San Antonio Oeste. Provincia de Río Negro. Instituto de Geología y Recursos Minerales, SEGEMAR, Boletín 254, Buenos Aires.

Meltzer, D. 1989. Was stone exchange among eastern North American paleoindians? En Eastern Paleoindian Lithic Resource Use, editado por C. Ellis y J. Lothrop, pp. 11-39. Westview Press, Boulder.

Monti, A.J. 2000. Edades ${ }^{14} \mathrm{C}$ y ciclicidad de la acreción en depósitos costeros elevados, Bahía Engaño, Chubut. Revista de la Asociación Geológica Argentina 55 (4):403-406.

Moore, D. 1995. Estadística Aplicada Básica. Antoni Bosch editor, Barcelona.

Pagès, J. 2002. Analyse factorielle multiple appliquée aux variables qualitatives et aux données mixtes. Statistique Appliqué 50 (4):5-37.

R Development Core Team 2005. R: A Language and Environment for Statistical Computing,Reference Index Version 2.2.1. R Foundation for Statistical Computing, Viena.

Scartascini, F. 2014. Arqueología de la Pesca en la Costa Rionegrina, Patagonia Argentina. Tesis Doctoral, Facultad de Filosofía y Letras, Universidad de Buenos Aires, Buenos Aires.

Schellmann, G. y T. Radtke 2010. Timing and magnitude of Holocene sea-level changes along the middle and south Patagonian Atlantic coast derived from beach ridge systems, littoral terraces and valley-mouth terraces. Earth-Science Reviews 103:1-30.

Shelley, P. 1993. A geoarchaeological approach to the analysis of secondary lithic deposits. Geoarchaeology: An International Journal 8 (1):59-72.

Torrence, R. 1983. Time budgeting and hunter-gatherer technology. En Hunter-Gatherer Economy in Prehistory: An European Perspective, editado por G. Bailey, pp. 11-22. Cambridge University Press, Cambridge. 